\title{
Combination therapies for primary hepatic neuroendocrine carcinoma: a case report
}

\author{
Richi Nakatake*, Morihiko Ishizaki, Kosuke Matui, Hiroaki Yanagimoto, Kentaro Inoue, Masaki Kaibori, \\ Yusai Kawaguchi and Masanori Kon
}

\begin{abstract}
Background: Primary hepatic neuroendocrine carcinomas are extremely rare. Because of the rarity of PHNEC, its clinical features and treatment outcomes are not well understood. A proper diagnosis and the correct therapeutic approach therefore remain clinically challenging.

Case presentation: A 67-year-old man was admitted to our department because of a liver tumor. Computed tomography revealed a single liver tumor $50 \mathrm{~mm}$ in diameter and located in the S3 region. Biopsy and imaging findings resulted in a diagnosis of primary hepatic neuroendocrine carcinoma. Left lateral segmentectomy was performed. Immunohistochemically, the tumor cells were positive for synaptophysin, chromogranin A, and CD56. Ki-67 was positive in $>90 \%$ of the tumor cells. The final diagnosis was primary hepatic neuroendocrine carcinoma. The patient suffered two episodes of lymph node recurrence. Nonetheless, the tumor was excised to prolong survival. Thus, after lymphadenectomy, he received adjuvant chemotherapy for 6 months. Two years after surgery, the patient remains alive and in good general condition.

Conclusions: In most cases, primary hepatic neuroendocrine carcinoma, while extremely rare, has a poor prognosis. At present, surgical resection is a priority for curative treatment, but in patients with recurrence, combined therapies are recommended.
\end{abstract}

Keywords: Primary hepatic neuroendocrine carcinoma, Liver resection, Hepatectomy

\section{Background}

Primary hepatic neuroendocrine carcinomas (PHNECs) are extremely rare, with roughly 64 cases reported in the English-language literature until 2016 [1-26]. Because of the rarity of PHNEC, its clinical features and treatment outcomes are not well understood. A proper diagnosis and the correct therapeutic approach therefore remain clinically challenging. We herein report a case of PHNEC.

\section{Case presentation}

A 67-year-old male was admitted to our hospital for evaluation and management of a symptomatic liver mass. His past medical history included bladder cancer for a postoperative follow-up. Liver dynamic computed tomography (CT) showed a low-density mass in the S3 area (Fig. 1a-c)

\footnotetext{
* Correspondence: nakatakr@hirakata.kmu.ac.jp

Department of Surgery, Kansai Medical University, 2-5-1 Shinmachi, Hirakata, Osaka 573-1010, Japan
}

(c) The Author(s). 2017 Open Access This article is distributed under the terms of the Creative Commons Attribution 4.0 International License (http://creativecommons.org/licenses/by/4.0/), which permits unrestricted use, distribution, and reproduction in any medium, provided you give appropriate credit to the original author(s) and the source, provide a link to the Creative Commons license, and indicate if changes were made. and magnetic resonance imaging (MRI) a mass with differraphy (PET)-CT, the SUV max of the tumor in S3 of the liver was 10 (Fig. 1e). Both MRI and PET-CT confirmed a single liver tumor $50 \mathrm{~mm}$ in diameter located in the S3 region. Because of the patient's past medical history, liver metastasis of bladder cancer or other cancers was suspected. Thus, we performed the liver biopsy preoperatively. The histological reports of biopsy revealed a solitary epithelial neoplasm. Immunohistochemistry demonstrated the expression of synaptophysin and CD56 (not chromogranin A). The patient was diagnosed with primary neuroendocrine carcinoma based on the biopsy results and imaging findings. To identify the primary neoplasm, chest and abdominal CT, upper and lower endoscopy, and abdominal MRI were performed. None of these examinations revealed a primary lesion outside the liver.

Upon presentation, the patient was afebrile, had no history of weight loss, and his appetite was good. His laboratory test results did not reveal any evidence of liver

\section{Springer Open}




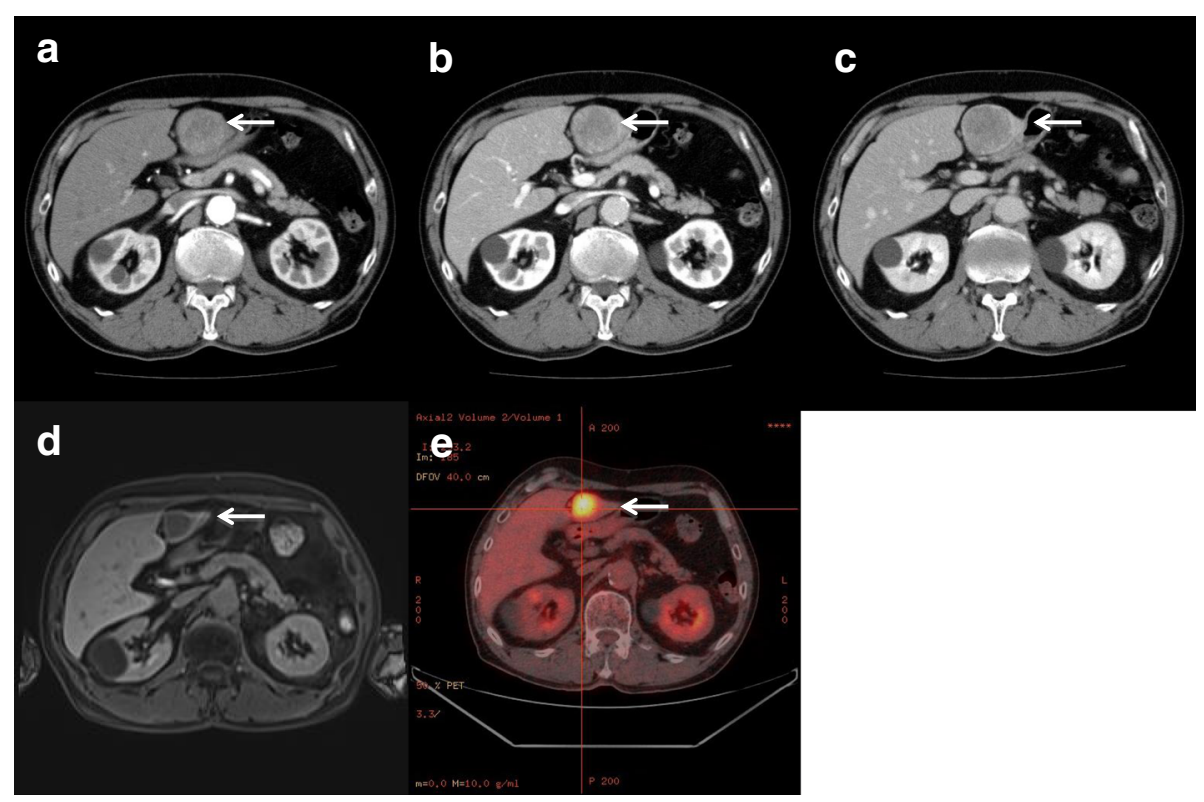

Fig. 1 a-c Liver dynamic computed tomography (CT). a Arterial phase; b Portal phase; c Delayed phase. The tumor shows low signal intensity, with an unclear border in each phase (arrows). $\mathbf{d}$ Gd-EOB-DTPA-MRI shows tumor nodules in the liver and a low-intensity and hypo-vascular tumor in segment 3 (arrow). e Positron emission tomography-CT. The SUV max of the tumor in S3 of the liver is 10 (arrow)

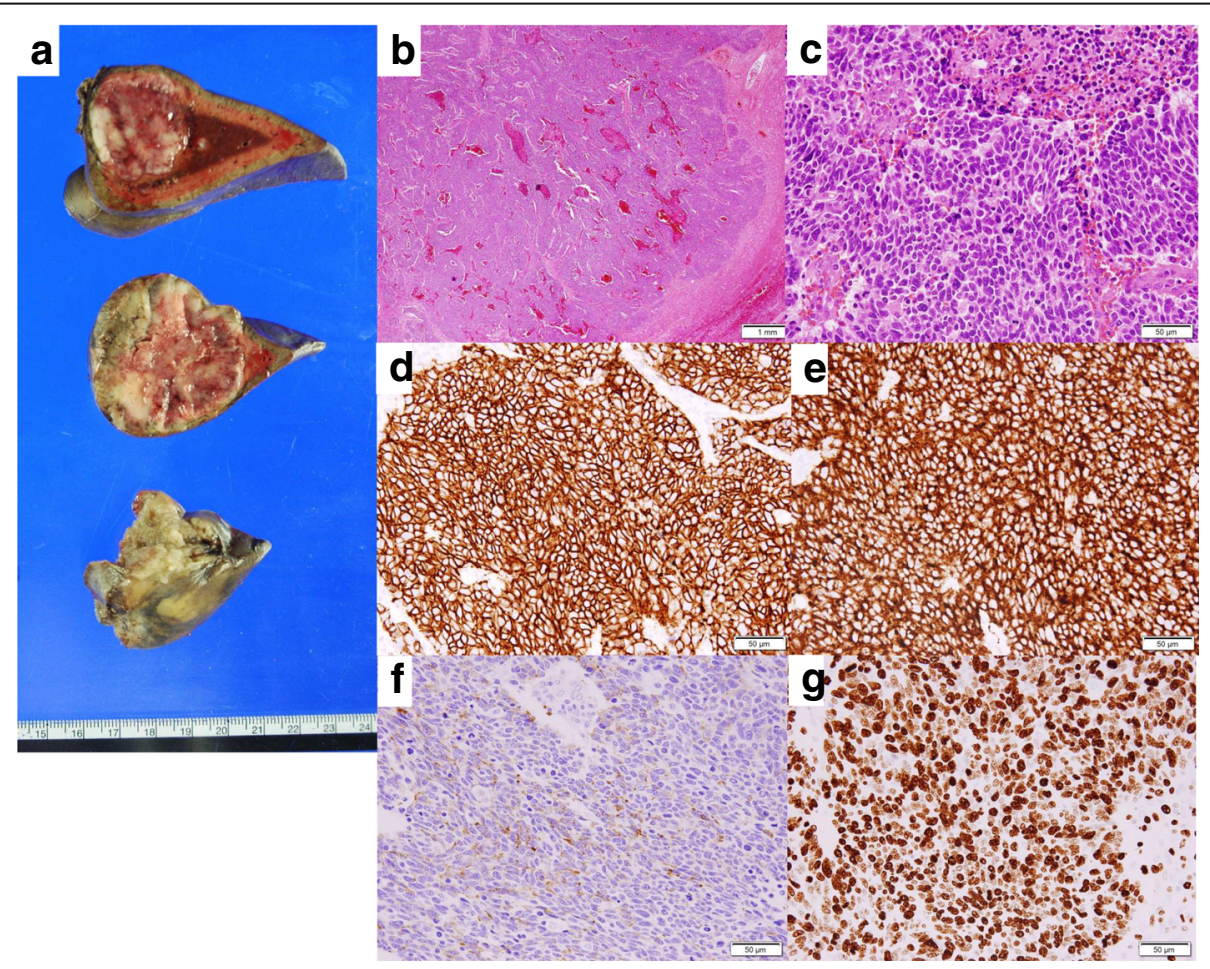

Fig. 2 Macroscopic and histopathological findings of the resected specimen. a The lateral segment consists of a solid mass. b, c Hematoxylin-eosin staining shows the enlarged nuclei and condensed chromatin of the tumor cells. $\mathbf{d}-\mathbf{g}$ Immunopathological examination. The tumor cells are positive for CD56 (d), synaptophysin (e), and chromogranin A (f). Ki-67 was positive in $>90 \%$ of the tumor cells ( $\mathbf{g})$ 
dysfunction. Antibodies against hepatitis B virus and hepatitis $C$ virus surface antigens were negative. Serum tumor markers alpha-fetoprotein, carcinoembryonic antigen, and cancer antigen 19-9 were within the normal range, but neuron-specific enolase levels increased $(27.3 \mathrm{mg} / \mathrm{dl})$. The patient was therefore diagnosed with primary liver cancer and a resection of the lateral segment of the liver was planned. After left lateral segmentectomy, the postoperative course was uneventful and the patient was discharged on the tenth postoperative day.

The pathology report revealed a solitary epithelial neoplasm with a well-developed vascular network (Fig. 2b, c) and no tumor invasion of vein and bile duct. The subclassification of NEC categorized into small type. The morphology of this tumor was homogeneous. There existed no components of adenocarcinoma and welldifferentiated NET. Immunohistochemistry demonstrated expression of synaptophysin, chromogranin A, and CD56. Ki-67 was positive in $>90 \%$ of the tumor cells (Fig. $2 d-$ g). The diagnosis of a primary neuroendocrine carcinoma of the liver was established (PHNEC grade 3). Resection margins were tumor-free ( $\mathrm{R} 0$ resection).

Three months after surgery, the patient suffered lymph node $(\# 3,8)$ recurrence (Fig. 3a, b) and underwent lymphadenectomy. Four months after the second surgery, recurrence was detected in the left renal vein lymph nodes (Fig. 3c, d). The tumor burden could be reduced using an antitumor agent (cisplatin + irinotecan) for 4 months to allow subsequent excision of the tumor. Thus, after lymphadenectomy, he received adjuvant chemotherapy (cisplatin + irinotecan) for 6 months. Two years after the first surgery, the patient remains in good general condition.

\section{Discussion}

In the fourth edition of the WHO Classification of Tumors of the Digestive System, published in 2010, the term "neuroendocrine neoplasms" replaced the previously used "neuroendocrine tumors" [27]. Neuroendocrine neoplasms can be categorized into three grade-based groups. Low- and intermediate-grade neuroendocrine neoplasms are neuroendocrine tumors grades 1 and 2, respectively; high-grade neuroendocrine neoplasms are neuroendocrine carcinomas. Neuroendocrine tumors develop in organs or tissues that contain peptide and amine-producing cells and exhibit different hormonal profiles depending on their site of origin $[28,29]$. Overall, approximately 57.0 and $27.0 \%$ of all neuroendocrine tumors arise within the gastroenteropancreatic and bronchopulmonary systems, respectively [30]. Within the gastrointestinal tract, most neuroendocrine tumors occur in the rectum (17.2\%), jejunum/ileum (13.4\%), and pancreas (6.4\%) [30].

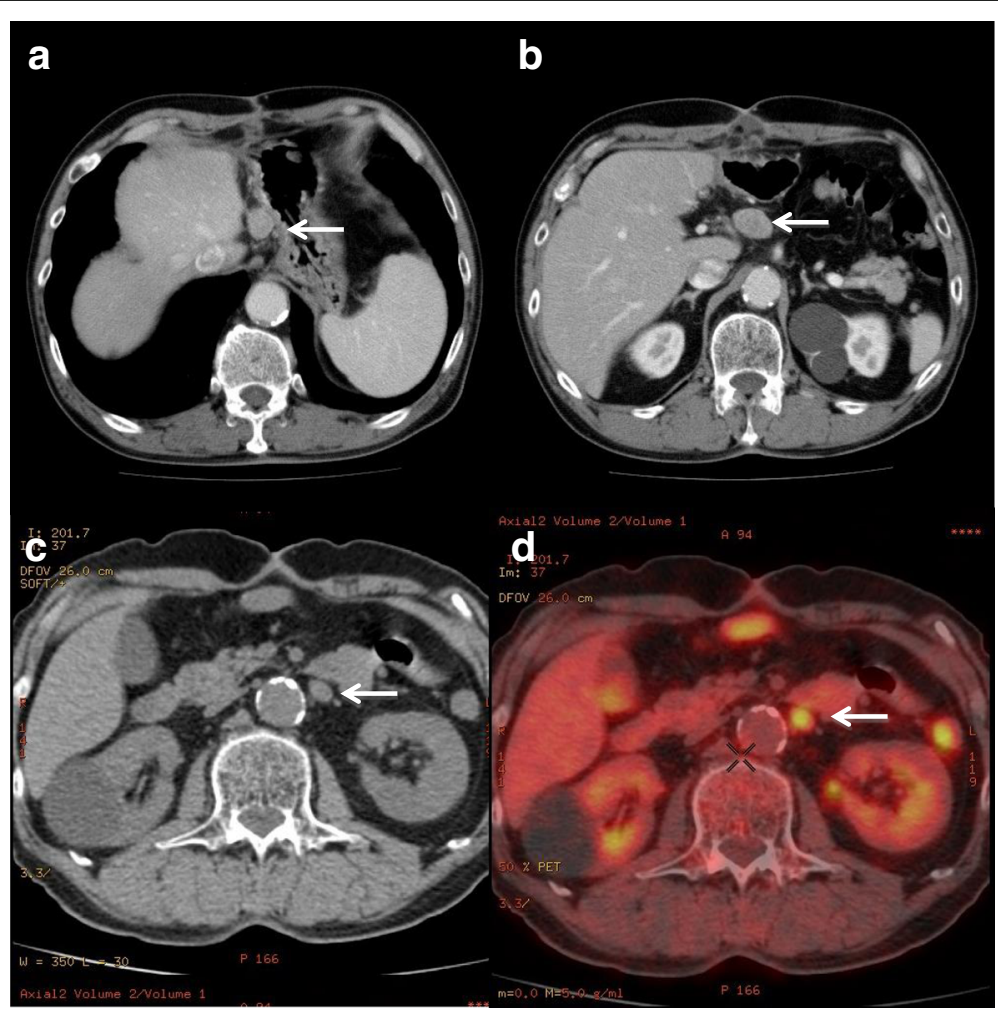

Fig. 3 a, b Computed tomography (CT) shows lymph node recurrence (a; \#3, b; \#8, arrows) . c, $\mathbf{d}$ CT and positron emission tomography-CT. c CT shows recurrence (arrow) in the left renal vein lymph nodes. d The SUV max of the tumor was 4.6 (arrow) 


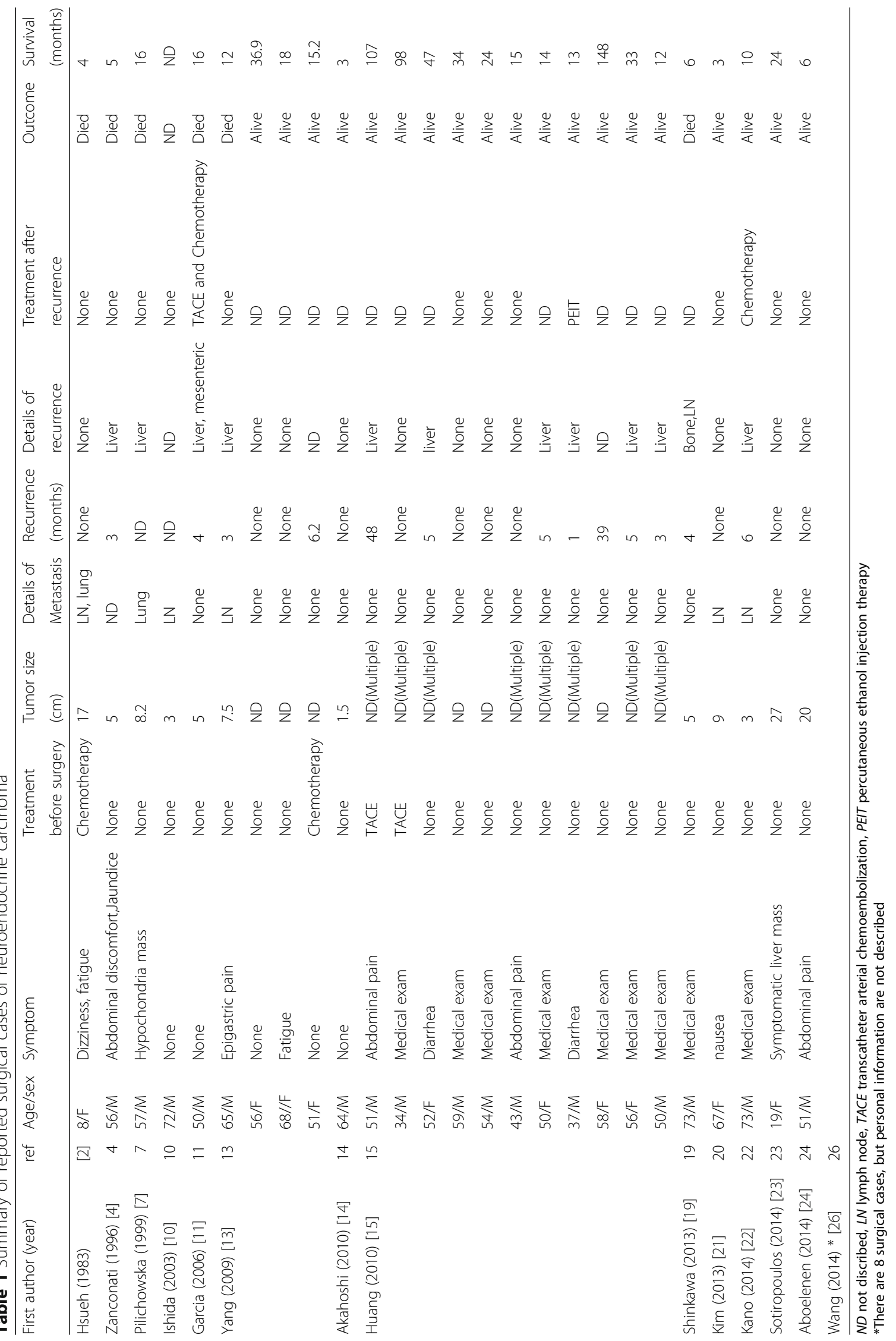


Neuroendocrine tumors are diagnosed by pathologic confirmation. On hematoxylin and eosin staining, the tumors may demonstrate an insular, trabecular, or glandular cell arrangement [7]. Immunohistochemical staining of these tumors reveals immunoreactivity to specific markers, including chromogranin A, neuron-specific enolase, and synaptophysin [31, 32]. The exclusion of an extrahepatic origin of the tumors and a pathological analysis of the neuroendocrine carcinoma are needed for the diagnosis of PHNEC. Because the liver is the most frequent metastatic site of neuroendocrine carcinoma, PHNEC must also be diagnostically differentiated from metastatic hepatic neuroendocrine carcinoma [14].

There is no standard for the therapy of PHNEC. Currently, surgery is the only curative option and provides the most favorable outcome, including long-term survival [15]. Only 33 reports of surgery in PHNEC patients have been published in the literature (Table 1) $[2,4,7,10,11,13-15$, 19, 20, 22-24, 26]. Park et al. reported on three patients with resectable tumors who were alive 17.7 months after treatment (range, 15.2-36.9 months) and on nine patients whose tumors could not be surgically removed but who survived for 11.3 months (range, 0.7-41.7 months) [20]. Even in a patient with a giant tumor, curative resection allowed long-term survival [23]. The preferred treatment for PHNEC for tumors without distant or lymph node metastasis is surgical resection [19]. Surgical resection for PHNEC is an independent predictor of survival. However, surgery alone is rarely curative, since the vast majority in patients with PHNEC undergoing resection will develop recurrences. It is a reason why adjuvant chemotherapy after curative resection should be considered, although no prospective studies are available to support this practice. While resection of all tumors could lead to a higher survival rate and better outcomes, many patients will still require combined therapy, such as transcatheter arterial chemoembolization, chemotherapy, and radiofrequency ablation [26].

There is still no report of typical treatment for recurrence in PHNEC. In Wang's series, combined therapy resulted in better outcomes than monotherapy even in patients with recurrence [26]. Tumor progression can be controllable with antitumor agents, and tumor burden can be reduced without the evidence of other recurrence, allowing subsequent excision in the tumor. Further studies are needed to more accurately determine the clinical features of PHNEC.

\section{Conclusions}

In conclusion, PHNEC is a rare liver primary tumor with unique specificity regarding its occurrence and development. At present, surgical resection is a priority for curative treatment, but in patients with recurrence, combined therapies are recommended.

\section{Abbreviations}

CT: Computed tomography; MRI: Magnetic resonance imaging; PHNEC: Primary hepatic neuroendocrine carcinoma

\section{Acknowledgements}

This study was not funded by any grants.

\section{Authors' contributions}

RN drafted the manuscript. MI has given the final approval of the version to be published. All authors read and approved the final manuscript.

\section{Consent for publication}

Written informed consent was obtained from the patient for publication of this case report and any accompanying images. A copy of the written consent is available for review by the Editor-in-Chief of this journal.

\section{Competing interests}

The authors declare that they have no competing interests.

\section{Publisher's Note}

Springer Nature remains neutral with regard to jurisdictional claims in published maps and institutional affiliations.

Received: 18 August 2017 Accepted: 5 September 2017

Published online: 11 September 2017

References

1. Xi YP, Yu JY. Primary neuroendocrine carcinoma of the liver. Ultrastruct Pathol. 1986;10(4):331-6.

2. Hsueh C, Tan XD, Gonzalez-Crussi F. Primary Hepatic Neuroendocrine Carcinoma in a Child. Cancer. 1993;71(8):2660-5.

3. Tsuchimochi S, Nakajo M, Inoue H, Miyazono N, Noguchi I, Matsushita T, et al. Primary hepatic neuroendocrine carcinoma with multiple metastases: a case report. Radiat Med. 1995;13(4):183-5.

4. Zanconati F, Falconieri G, Lamovec J, Zidar A. Small cell carcinoma of the liver: a hitherto unreported variant of hepatocellular carcinoma. Histopathology. 1996;29(5):449-53.

5. Fukunaga M. Neuroendocrine carcinoma of the liver: an autopsy case. Pathol Int. 1998;48(6):481-5.

6. Ferrero A, Gallino C, D'Aloisio G, Gandini G, Garavoglia M. Primary neuroendocrine carcinoma of the liver: difficult diagnosis of a rare neoplasm. Acta Chir Belg. 1999;99(6):299-302.

7. Pilichowska M, Kimura N, Ouchi A, Lin H, Mizuno Y, Nagura H. Primary hepatic carcinoid and neuroendocrine carcinoma: clinicopathological and immunohistochemical study of five cases. Pathol Int. 1999;49(4):318-24.

8. Kaya G, Pasche C, Osterheld MC, Chaubert P, Fontolliet C. Primary neuroendocrine carcinoma of the liver: an autopsy case. Pathol Int. 2001; 51(11):874-8

9. Ishizu A, Yokoyama K, Yoshiki T. Primary neuroendocrine carcinoma of the liver diagnosed at autopsy. J Gastroenterol Hepatol. 2003;18(8):1002-4.

10. Ishida M, Seki K, Tatsuzawa A, Katayama K, Hirose K, Azuma T, et al. Primary hepatic neuroendocrine carcinoma coexisting with hepatocellular carcinoma in hepatitis C liver cirrhosis: report of a case. Surg Today. 2003; 33(3):214-8.

11. Garcia MT, Bejarano PA, Yssa M, Buitrago E, Livingstone A. Tumor of the liver (hepatocellular and high grade neuroendocrine carcinoma): a case report and review of the literature. Virchows Arch. 2006:449(3):376-81.

12. Yasuda E, Takeshita A, Murata S, Ihaku Y, Nitta T, Akutagawa H, et al. Neuroendocrine carcinoma of the liver associated with dermatomyositis: autopsy case and review of the literature. Pathol Int. 2006;56(12):749-54.

13. Yang CS, Wen MC, Jan YJ, Wang J, Wu CC. Combined primary neuroendocrine carcinoma and hepatocellular carcinoma of the liver. J Chin Med Assoc. 2009;72(8):430-3.

14. Akahoshi T, Higashi H, Tsuruta S, Tahara K, Matsumoto T, Takeuchi H, et al. Primary neuroendocrine carcinoma coexisting with hemangioma in the liver: report of a case. Surg Today. 2010;40(2):185-9.

15. Huang YQ, Xu F, Yang JM, Huang B. Primary hepatic neuroendocrine carcinoma: clinical analysis of 11 cases. Hepatobiliary Pancreat Dis Int. 2010; 9(1):44-8.

16. Iwasa S, Morizane C, Okusaka T, Ueno H, Ikeda M, Kondo S, et al. Cisplatin and etoposide as first-line chemotherapy for poorly differentiated 
neuroendocrine carcinoma of the hepatobiliary tract and pancreas. Jpn J Clin Oncol. 2010;40(4):313-8.

17. Takeuchi S, Honma R, Taguchi J, Amano T, Shimizu Y, Kinoshita I, et al. A case of high-grade neuroendocrine carcinoma that improved with Bevacizumab plus modified FOLFOX6 as the fourth-line chemotherapy. Case Rep Oncol. 2011;4(2):260-6.

18. Alekseev D, Goralczyk A, Lorf T, Ramadori G, Obed A. Ten years survival with excellent outcome after living donor liver transplantation from 70 years old donor for primary hepatic neuroendocrine carcinoma: case report. Int J Surg Case Rep. 2012;3(1):34-6.

19. Shinkawa H, Takatsuka S, Kaizaki R, Fujiwara Y, Kurai O, Yamazaki O. Postoperative outcomes of primary hepatic neuroendocrine carcinomas: review article. Osaka City Med J. 2013;59(2):105-13.

20. Park CH, Chung JW, Jang SJ, Chung MJ, Bang S, Park SW, et al. Clinical features and outcomes of primary hepatic neuroendocrine carcinomas. J Gastroenterol Hepatol. 2012;27(8):1306-11.

21. Kim JM, Kim SY, Kwon CH, Joh JW, Park JB, Lee JH, et al. Primary hepatic neuroendocrine carcinoma. Korean J Hepatobiliary Pancreat Surg. 2013; 17(1):34-7.

22. Kano Y, Kakinuma S, Goto F, Azuma S, Nishimura-Sakurai Y, Itsui Y, et al. Primary hepatic neuroendocrine carcinoma with a cholangiocellular carcinoma component in one nodule. Clin J Gastroenterol. 2014;7(5):449-54.

23. Sotiropoulos GC, Charalampoudis P, Delladetsima I, Stamopoulos P, Dourakis $S$, Kouraklis G. Surgery for giant primary neuroendocrine carcinoma of the liver. J Gastrointest Surg. 2014;18(4):839-41.

24. Aboelenen A, El-Hawary AK, Megahed N, Zalata KR, El-Salk EM, Fattah MA, et al. Right hepatectomy for combined primary neuroendocrine and hepatocellular carcinoma. A case report. Int J Surg Case Rep. 2014;5(1):26-9.

25. Huang J, Yu JQ, Sun JY. Computer tomography and magnetic resonance image manifestations of primary hepatic neuroendocrine cell carcinomas. Asian Pac J Cancer Prev. 2014;15(6):2759-64.

26. Wang $L M, A n S L$, Wu JX. Diagnosis and therapy of primary hepatic neuroendocrine carcinoma: clinical analysis of 10 cases. Asian Pac J Cancer Prev. 2014;15:2541-6.

27 Bosman FT, Carmeiro F, Hruban RH, Theise ND. WHO classification of tumours of the digestive system. Lyon International Agency for Research on Cancer. 2010.

28 Klöppel G, Heitz PU. Classification of normal and neoplastic neuroendocrine cells. Ann N Y Acad Sci. 1994;733:19-23.

29 Klöppel G, Perren A, Heitz PU. The gastroenteropancreatic neuroendocrine cell system and its tumors: the WHO classification. Ann N Y Acad Sci. 2004 1014:13-27.

30 Yao JC, Hassan M, Phan A, Dagohoy C, Leary C, Mares JE, et al. One hundred years after "carcinoid": epidemiology of and prognostic factors for neuroendocrine tumors in 35,825 cases in the United States. J Clin Oncol. 2008:26(18):3063-72.

31 Iwao M, Nakamuta M, Enjoji M, Kubo H, Fukutomi T, Tanabe Y, et al. Primary hepatic carcinoid tumor: case report and review of 53 cases. Med Sci Monit. 2001;7(4):746-50

32 Fenoglio LM, Severini S, Ferrigno D, Gollè G, Serraino C, Bracco C, et al. Primary hepatic carcinoid: a case report and literature review. World J Gastroenterol. 2009;15(19):2418-22.

\section{Submit your manuscript to a SpringerOpen ${ }^{\circ}$ journal and benefit from:}

- Convenient online submission

- Rigorous peer review

- Open access: articles freely available online

- High visibility within the field

- Retaining the copyright to your article 